\title{
Relationship Between Mobility and Self-Care Activity in Children With Cerebral Palsy
}

\author{
Kyeongwon Kim, $\mathrm{MD}^{1}$, Jin Young Kang, MD, PhD², Dae-Hyun Jang, MD, PhD ${ }^{1}$ \\ 'Department of Rehabilitation Medicine, College of Medicine, The Catholic University of Korea, Seoul; \\ ${ }^{2}$ Seoul Asan Rehabilitation Clinic, Incheon, Korea
}

\begin{abstract}
Objective To investigate the factors influencing the development of self-care activity, and the association between mobility and self-care activity in children with cerebral palsy (CP).

Methods A total of $63 \mathrm{CP}$ children aged $\geq 4$ years, were studied retrospectively. Children with severe intellectual disability or behavioral problems were excluded. The relationship between the Gross Motor Function Classification System (GMFCS), the Manual Ability Classification System (MACS), and the Pediatric Evaluation of Disability Inventory (PEDI) was analyzed. Simple and multiple linear regression analyses were conducted for continuous variables, such as verbal intelligence quotient (IQ) and PEDI subscales.

Results Final evaluation was done for 25 children, ranging from 4 to 11 years of age. According to GMFCS levels, the differences in PEDI-self-care scores, showed statistically borderline significance $(p=0.051)$. Conversely, differences in PEDI-self-care scores according to CP types and MACS levels were not statistically significant. Simple linear regression analysis showed that PEDI mobility and PEDI social function significantly influence the PEDI self-care. Multiple linear regression analysis showed that PEDI mobility was the only factor significantly influencing PEDI self-care in children aged $\geq 7$ years $\left(\mathrm{R}^{2}=0.875, \mathrm{p}=0.03\right)$.

Conclusion Mobility is important for the acquisition of self-care abilities in children with $\mathrm{CP}$ aged $\geq 7$ years.
\end{abstract}

Keywords Cerebral palsy, Activities of daily living, Self care, Mobility limitation

\section{INTRODUCTION}

Tasks required for personal care, such as feeding, personal hygiene, dressing, toileting, and functional mobility, comprise the activities of daily living (ADL). Instrumental activities of daily living (I-ADL) refer to the more comprehensive activities required for independent life, including social tasks (e.g., communication) and community mobility (e.g., taking public transportation) [1]. Methods for assessing daily activities encompassing ADL and I-ADL in cerebral palsy (CP) children led to investigations of the age-associated development of gross

Received May 24, 2016; Accepted August 31, 2016

Corresponding author: Dae-Hyun Jang

Department of Rehabilitation Medicine, Incheon St Mary's Hospital, College of Medicine, The Catholic University of Korea, 56, Dongsu-ro, Bupyeonggu, Incheon 21431, Korea. Tel: +82-32-280-5207, Fax: +82-32-280-5040, E-mail: dhjangmd@naver.com

ORCID: Kyeongwon Kim (http://orcid.org/0000-0001-6185-0058); Jin Young Kang (http://orcid.org/0000-0002-5262-2712); Dae-Hyun Jang (http:// orcid.org/0000-0001-8293-084X).

(c) This is an open-access article distributed under the terms of the Creative Commons Attribution Non-Commercial License (http://creativecommons.org/ licenses/by-nc/4.0) which permits unrestricted noncommercial use, distribution, and reproduction in any medium, provided the original work is properly cited. Copyright (c) 2017 by Korean Academy of Rehabilitation Medicine 
motor capacity, mobility, and other factors influencing this development [2-5]. For prompt evaluation and treatment, the daily activities of disabled children have been conceptualized using three domains: mobility, self-care, and social function [6].

Children acquire independence in daily living by improving their gross motor capacity, as well as manual, intellectual, and communicative functioning. Ability to perform gross motor function affects various types of daily activities, and is considered to be a predictor of selfcare activities and mobility [7]. Gross motor capacity differs from mobility and refers to an underlying ability to perform a task in a standardized environment. While the gross motor capacity is related to a quantified ability and functional limitations in a specific standardized environment, mobility correlates to the qualified ability of motor performance that is actually performed in a daily life, coinciding with the concepts of 'capacity,' 'capability', and 'performance' [8].

Self-care activities for infants and small children are performed by their caregivers/parents. Gradual independence is achieved in these activities by children, based on various intrinsic and extrinsic factors, including intellectual, personal and hand function development. Despite sufficient gross motor, intellectual and manual abilities, some children are unable to achieve independence in self-care activities. This is especially applicable in children with $\mathrm{CP}$, where a lack of independence in daily activities may be due to their dependence on assistance for mobility. We believe that children with CP who walk independently, with or without the aid of walking devices, achieve greater independence in self-care than children who are dependent on others for mobility. CP children assisted by their caregivers/parents for walking, may be given further assistance for other daily self-care activities unrelated to mobility and trunk stability, including help in washing hands, brushing their hair and teeth, and using utensils for feeding. Thus, independent mobility may affect independence in self-care activities. Accordingly, we investigated the factors associated with the development of self-care activity, and the relationship between mobility and self-care activities in children with CP.

\section{MATERIALS AND METHODS}

This retrospective study included children with CP who visited Incheon St. Mary's hospital from May 2015 to April 2016. The inclusion criteria were: (1) children diagnosed with CP; (2) children between the ages of 4 and 18 years; (3) children who had been evaluated by the Manual Ability Classification System (MACS), Gross Motor Function Classification System (GMFCS), Korean-Wechsler Intelligence Scale for Children (K-WISC) or Korean-Wechsler Preschool and Primary Scale of Intelligence (K-WPPSIIII), and Pediatric Evaluation of Disability Inventory (PEDI). Exclusion criteria included: (1) severe intellectual disability, according to verbal intelligence quotient (IQ) $<35$; (2) behavioral problems; and (3) hearing or visual impairment.

The PEDI, which assesses the functional skills for selfcare, mobility, and social function, examines the tasks that children are able to perform in the context of their daily lives (activity). Scaled scores on the PEDI reflect the functional skills of a child, scored from 0 to 100, where 0 represents no ability and 100 represents full capability to perform the functional skill items in each domain [6].

The MACS evaluates the self-initiated manual ability of a child and the need for assistance or adaptation in handling objects in daily life. The validity and reliability of MACS classification has been well-established, and ranges from level I (the child handles most objects), to level V (the child cannot handle any object) for children aged 4 to 18 years [9]. Korean versions of PEDI and MACS are widely used, both of which have also been shown to be valid and reliable $[10,11]$.

The GMFCS classifies the gross motor function of a child onto one of five levels: from level I, where the child walks without limitations, to level V, where self-mobility is severely limited, even requiring assistance [12].

Intelligence was evaluated in children aged $<6$ years using the K-WPPSI-III, and in children aged $\geq 6$ years using the Wechsler Intelligence Scale for Children-Revised (WISC-R, Korean version). As impairment in motor and coordination in children with $\mathrm{CP}$ attributes to a low performance IQ, we determined the severity of intellectual disability by verbal IQ, which more specifically represents the cognitive function in children with $\mathrm{CP}$. As severe intellectual disability can lead to an inability to perform self-care activities, children with verbal IQ $<35$ were excluded from this study.

This study was approved by Institutional Review Board of Incheon St. Mary's hospital. 


\section{Statistical analysis}

All statistical analyses were performed using SPSS ver. 18.0 (SPSS Inc., Chicago, IL, USA), with p-values $<0.05$ considered to be statistically significant. The relationship between the distribution of MACS and GMFCS scores were analyzed using Spearman rank correlation coefficient. The relationship between MACS, GMFCS scores and CP type with PEDI self-care scores were analyzed using the Kruskal-Wallis test. To investigate the factors influencing PEDI self-care scores, continuous variables such as verbal IQ, PEDI mobility and PEDI social function scores were analyzed using simple and multiple linear regression tests. The PEDI self-care scores were first analyzed by simple linear regression tests, after which the significant factors from the simple linear regression analysis were entered into multiple linear regression analysis.

Table 1. Demographic and clinical characteristics of the 25 subjects enrolled in this study

\begin{tabular}{|cc|}
\hline \multicolumn{1}{|c}{ Characteristic } & Value \\
\hline Sex & 11 \\
\hline Male & 14 \\
\hline Female & $6 ; 0 \pm 2 ; 0(4 ; 2-11 ; 4)$ \\
\hline Age (yr;mo) & \\
\hline IQ & $83.0 \pm 16.5$ \\
\hline Verbal IQ & $78.0 \pm 12.7$ \\
\hline Performance IQ & $78.9 \pm 15.2$ \\
\hline Full scale IQ & \\
\hline Type of cerebral palsy & 8 \\
\hline Spastic unilateral & 16 \\
\hline Spastic bilateral & 1 \\
\hline Ataxic & \\
\hline GMFCS & $13 / 5 / 3 / 2 / 2$ \\
\hline I/II/III/IV/V & \\
\hline MACS & $5 / 9 / 8 / 2 / 1$ \\
\hline I/II/III/IV/V & \\
\hline PEDI & $66.0 \pm 14.3$ \\
\hline Self-care & $76.8 \pm 13.8$ \\
\hline Mobility & $71.7 \pm 16.1$ \\
\hline Social function & \\
\hline
\end{tabular}

Values are presented as number or mean \pm standard deviation (range).

IQ, intellectual quotient; GMFCS, Gross Motor Function Classification System; MACS, Manual Ability Classification System; PEDI, Pediatric Evaluation of Disability Inventory.

\section{RESULTS}

From the $63 \mathrm{CP}$ children screened for this study, 38 were excluded because of incomplete data or meeting exclusion criteria described earlier. Finally, 25 children (11 boys, 14 girls) with mean age of 6 years, were enrolled in our study. There were 8 cases (32\%) of spastic unilateral type $\mathrm{CP}, 16$ cases $(64 \%)$ of spastic bilateral type $\mathrm{CP}$, and 1 case $(4 \%)$ of ataxic type CP (Table 1). The distribution of MACS scores was significantly associated with the severity of gross motor deficits $(\rho=0.437, p=0.029)$ (Fig. 1$)$.

Mean PEDI self-care was higher in children with spastic unilateral type than in other types of $\mathrm{CP}$, in children with a GMFCS level of I compared to GMFCS levels of II-V, and in children with a MACS level of I compared to MACS levels of II-V. The differences in the PEDI-self-care scores according to GMFCS levels were shown to be of statistically borderline significance $(\mathrm{p}=0.051)$. Conversely, differences in PEDI-self-care scores according to CP types and MACS levels were not statistically significant (Table 2).

Simple linear regression analysis revealed that PEDI mobility and PEDI social function significantly influence PEDI self-care (Table 3). Previous studies have reported that gross motor skills plateau at age 7 in children with CP $[5,13,14]$. We therefore performed linear regression analysis in children aged $<7$ and children aged $\geq 7$ years. Simple regression analysis showed that PEDI mobility and PEDI social function significantly influence PEDI self-care in both groups, aged $<7$ and aged $\geq 7$ years. Mul-

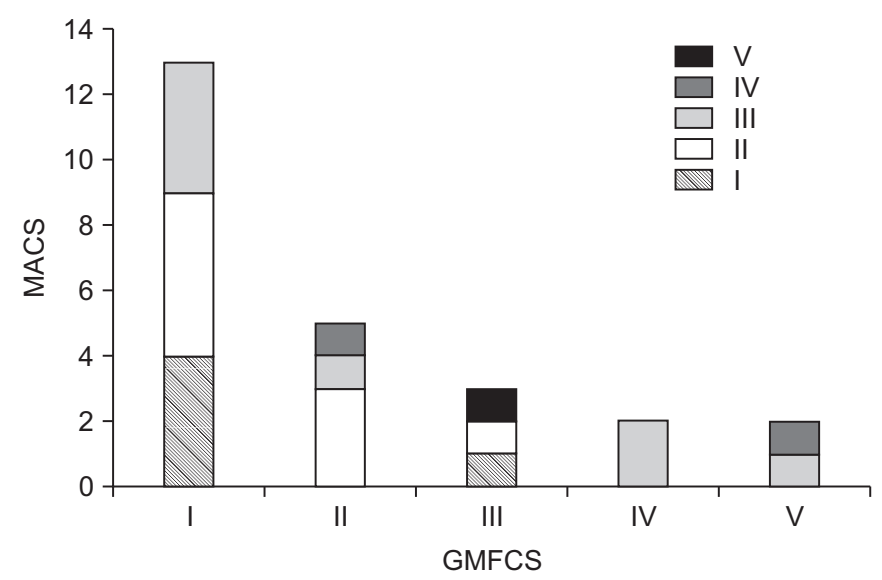

Fig. 1. The distribution of hand function is significantly associated with the severity of gross motor function. GMFCS, Gross Motor Function Classification System; MACS, Manual Ability Classification System. 
Table 2. PEDI self-care scores according to the type of CP, GMFCS and MACS classifications

\begin{tabular}{|c|c|c|}
\hline & PEDI self-care & p-value \\
\hline Type of CP & & 0.13 \\
\hline Spastic unilateral & $73.9 \pm 9.4$ & \\
\hline Bilateral & $62.4 \pm 15.4$ & \\
\hline Ataxic & $59.9 \pm 0.0$ & \\
\hline GMFCS & & 0.05 \\
\hline I & $72.7 \pm 10.0$ & \\
\hline II & $67.7 \pm 6.6$ & \\
\hline III & $59.7 \pm 19.3$ & \\
\hline IV & $59.1 \pm 1.2$ & \\
\hline V & $34.7 \pm 6.0$ & \\
\hline MACS & & 0.07 \\
\hline I & $76.5 \pm 6.4$ & \\
\hline II & $68.4 \pm 10.7$ & \\
\hline III & $64.9 \pm 12.9$ & \\
\hline IV & $47.2 \pm 23.7$ & \\
\hline V & $37.4 \pm 0.0$ & \\
\hline
\end{tabular}

Values are presented as mean \pm standard deviation.

CP, cerebral palsy; GMFCS, Gross Motor Function Classification System; MACS, Manual Ability Classification System; PEDI, Pediatric Evaluation of Disability Inventory. tiple regression analysis was performed using PEDI mobility and PEDI social function as independent variables in both groups (Table 4 ). In children aged $\geq 7$ years, PEDI mobility was the only significant factor explaining PEDI self-care. In children aged $<7$ years, no significant factor was identified.

\section{DISCUSSION}

This study investigated factors that were associated with self-care activities and found that mobility is a significant factor in self-care activities of CP children aged $\geq 7$ years.

As low distinguishability of self-care ability in children with severe intellectual disability was presumed by the 'floor effect', the CP children with verbal IQ $<35$ were excluded. Consequently, a majority of children with GMFCS V and MACS V were excluded. The levels of GMFCS and MACS in children included the study were mainly distributed in the levels of I-III.

In our study, the influence of gross motor ability on self-care activity was not strongly significant but only shown to be borderline. The influence of hand function on self-care activities was also not significant. To date, few studies that have previously sought to assess the re-

Table 3. Simple linear regression analysis of factors influencing PEDI self-care

\begin{tabular}{lcccc}
\hline & \multicolumn{4}{c}{ Simple linear regression test } \\
\cline { 2 - 5 } & $\boldsymbol{\beta}$ & SE $(\beta)$ & $\mathbf{R}^{2}$ & p-value \\
\hline Verbal IQ & 0.19 & 0.18 & 0.048 & 0.29 \\
PEDI mobility & 0.64 & 0.17 & 0.376 & $0.001^{*}$ \\
PEDI social function & 0.56 & 0.15 & 0.392 & $0.001^{*}$ \\
\hline
\end{tabular}

IQ, intellectual quotient; PEDI, Pediatric Evaluation of Disability Inventory. ${ }^{*} \mathrm{p}<0.05$.

Table 4. Simple and multiple linear regression tests of factors influencing PEDI self-care in children aged $<7$ and $\geq 7$ years

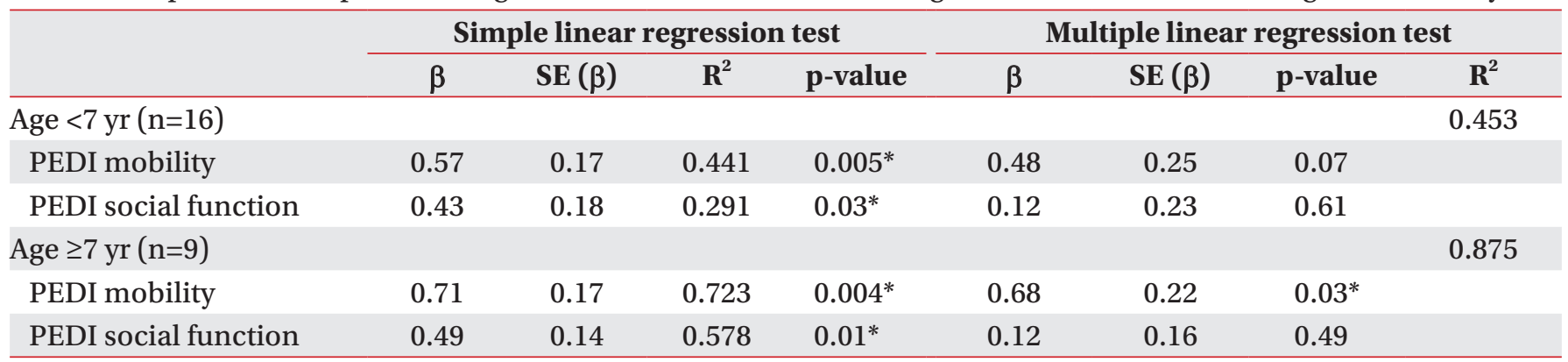

PEDI, Pediatric Evaluation of Disability Inventory. ${ }^{*} \mathrm{p}<0.05$. 
lationship between gross motor abilities and daily life activities in children with $\mathrm{CP}$, have all reported that gross motor capacity is strongly associated with everyday functioning $[3,7,15,16]$. Previous researches also state that the relationships between hand function and self-care activities are significant $[4,17,18]$. The differing results of our study, in contrast to the previous studies, could be due to the difference of inclusion criteria. Since our research included CP children with IQ $\geq 35$ with majority of children in levels I-III by GMFCS and MACS, hence the difference in results deduced. The results of our study implies that self-care activity may not be critically limited by gross motor function and hand function in children with mild to moderate cerebral palsy.

Simple linear regression analysis of our study showed that independence in self-care is not influenced by verbal IQ. Although it had been posited that self-care activities require appropriate cognitive functions, we found that PEDI self-care did not correlate with verbal IQ in children with $\mathrm{CP}$, after excluding children with the most severe disabilities in intellectual capacity (verbal IQ $<35$ ) $[3,19]$. This result implies that performing self-care activities does not require high intellectual ability, and children with mild to moderate intellectual disability have sufficient potential for performing self-care activities.

None of the previous studies assessed the relationship between mobility and self-care activity. Although gross motor capacity is considered an important factor for mobility in daily life, the correlation between gross motor capacity and mobility is difficult to assess. Both the concept of gross motor capacity and the concept of dailylife mobility are found within the 'activities' domain of the International Classification of Functioning, Disability and Health [20]. Gross motor capacity is unrelated to daily-life mobility in children with unilateral spastic CP [2]. Therefore, we defined mobility as performance, i.e., what an individual actually does in a daily environment, and not as a gross motor capacity [8]. Accordingly, we used the PEDI mobility score to reflect the specific environment. The PEDI mobility domain includes getting in and out of a chair/wheelchair, car, bed, bathtub, and toilet seat, in addition to the method and quality of indoor/ outdoor locomotion, and going upstairs and downstairs. The PEDI self-care domain includes factors such as food texture, nose care, hair brushing, teeth cleaning, washing the body and face, dressing, use of the toilet, bowel and bladder management, and use of utensils and drinking containers. As shown by these sub-questions, PEDI mobility and PEDI self-care domains individually assess different aspects of everyday functionality in children with cerebral palsy [15]. Thus, PEDI self-care domain itself is not directly related to the mobility domain.

Although mobility and social function are significant factors influencing self-care activity by simple linear regression analysis, multiple linear regression analysis revealed that mobility was the only factor influencing self-care activity in children aged $\geq 7$ years. A longitudinal study of children with disabilities found that motherchild interactions and child self-regulatory processes (mastery of motivation and behavior) were key predictors of changes in developmental outcomes [21]. Under certain specific conditions, the parents of children with restricted mobility help their children perform self-care activities unrelated to mobility irrespective whether the child has sufficient fine motor skills, thus altering the child's mastery of motivation or family ecology. Hence, dependence on others for mobility leads to dependence on others for self-care activity. On the other hand, Herbert et al. [22] reported that independent locomotion was associated with the ability of infants to retrieve memories. Crawling infants exhibited better memory retrieval when given different stimuli under different contexts, as compared to non-crawling infants. It has been suggested that independent locomotion provides varied experience, which in turn leads to greater flexible memory retrieval in infants. As flexible memory retrieval is a key component of highly sophisticated cognitive skills crucial for daily life, improving the flexible memory retrieval can lead to better self-care abilities in adults as well as in children. In visuospatial processing, the dorsal visuospatial pathway, including networks of the dorsal parietal lobe and the superior frontal cortex, play a key role in controlling reactive body movement based on visual information [23]. When given visual stimuli, the prefrontal cortex begins to plan and prepare a specific movement and the dorsal parietal lobe relays this signal to the premotor and motor cortex to initiate body movement. Various visual stimuli provided by independent mobility activate areas of the brain, including the frontal and superior parietal cortex, thereby improving the key elements of self-care activities-the cognitive skills and spatial perception.

Previous studies have reported that gross motor func- 
tion curves plateau at 7 years of age $[5,13,14]$. These findings, along with our results, suggest that mobility development rather than gross motor development is important in developing self-care activity in children aged $\geq 7$ years old. Hence, a rehabilitation program aimed at improving mobility is crucial in children with CP aged $\geq 7$ years, since it forms the basis for further improvements in self-care activity, leading to significant improvements in the quality of life.

This study had several limitations, including its retrospective design and small sample size. In this single center study, we were unable to recruit more patients meeting the inclusion criteria (aged $\geq 4$ years, evaluated by PEDI, GMFCS, MACS and K-WPPSI-III or K-WISC, excluding severe intellectual disability and incomplete data). Further researches conducted in multi-centers with larger number of subjects are needed to reliably investigate the influence of mobility and gross motor abilities on self-care activities. Additionally, we believe that for future studies, a registration system for children with CP would enable a multi-center study enrolling greater number of subjects. Another limitation was that the majority of children enrolled in this study had mild gross motor impairment with GMFCS level I or II, which could influence our results, and therefore should be considered in interpretation. Moreover, the effects of adaptive behavior and the environment, including factors such as culture, were not assessed.

In conclusion, in CP children aged $\geq 7$ years, mobility was the only significant factor influencing independence of self-care activities. These results may be clinically applicable in setting realistic treatment plans for improving their development.

\section{CONFLICT OF INTEREST}

No potential conflict of interest relevant to this article was reported.

\section{REFERENCES}

1. American Occupational Therapy Association. Occupational therapy practice framework: domain and process. Am J Occup Ther 2002;56:609-39.

2. Smits DW, Gorter JW, Ketelaar M, Van Schie PE, Dallmeijer AJ, Lindeman E, et al. Relationship between gross motor capacity and daily-life mobility in children with cerebral palsy. Dev Med Child Neurol 2010; 52:e60-6.

3. Smits DW, Ketelaar M, Gorter JW, van Schie P, Dallmeijer A, Jongmans M, et al. Development of daily activities in school-age children with cerebral palsy. Res Dev Disabil 2011;32:222-34.

4. Ohrvall AM, Eliasson AC, Lowing K, Odman P, Krumlinde-Sundholm L. Self-care and mobility skills in children with cerebral palsy, related to their manual ability and gross motor function classifications. Dev Med Child Neurol 2010;52:1048-55.

5. Hanna SE, Rosenbaum PL, Bartlett DJ, Palisano RJ, Walter SD, Avery L, et al. Stability and decline in gross motor function among children and youth with cerebral palsy aged 2 to 21 years. Dev Med Child Neurol 2009;51:295-302.

6. Haley SM, Coster WJ, Ludlow LH, Haltiwanger JT, Andrellos PJ. Pediatric Evaluation of Disability Inventory (PEDI): development, standardization, and administration manual. Boston: PEDI Resarch Group; 1992.

7. Voorman JM, Dallmeijer AJ, Schuengel C, Knol DL, Lankhorst GJ, Becher JG. Activities and participation of 9- to 13-year-old children with cerebral palsy. Clin Rehabil 2006;20:937-48.

8. Holsbeeke L, Ketelaar M, Schoemaker MM, Gorter JW. Capacity, capability, and performance: different constructs or three of a kind? Arch Phys Med Rehabil 2009;90:849-55.

9. Eliasson AC, Krumlinde-Sundholm L, Rosblad B, Beckung E, Arner M, Ohrvall AM, et al. The Manual Ability Classification System (MACS) for children with cerebral palsy: scale development and evidence of validity and reliability. Dev Med Child Neurol 2006; 48:549-54.

10. Kim WH, Park EY, Park SY. Reliability and validity of the Korean translation of the pediatric evaluation of disability inventory in school-aged children with cerebral palsy. Phys Ther Korea 2010;17:69-76.

11. Jang DH, Sung IY, Kang JY, Lee SI, Park JY, Yuk JS, et al. Reliability and validity of the Korean version of the manual ability classification system for children with cerebral palsy. Child Care Health Dev 2013;39:90-3.

12. Palisano RJ, Rosenbaum P, Bartlett D, Livingston MH. Content validity of the expanded and revised Gross Motor Function Classification System. Dev Med Child 
Neurol 2008;50:744-50.

13. Rosenbaum PL, Walter SD, Hanna SE, Palisano RJ, Russell DJ, Raina P, et al. Prognosis for gross motor function in cerebral palsy: creation of motor development curves. JAMA 2002;288:1357-63.

14. Smits DW, Gorter JW, Hanna SE, Dallmeijer AJ, van Eck M, Roebroeck ME, et al. Longitudinal development of gross motor function among Dutch children and young adults with cerebral palsy: an investigation of motor growth curves. Dev Med Child Neurol 2013;55:378-84.

15. Ostensjo S, Carlberg EB, Vollestad NK. Everyday functioning in young children with cerebral palsy: functional skills, caregiver assistance, and modifications of the environment. Dev Med Child Neurol 2003;45:60312.

16. Ostensjo S, Carlberg EB, Vøllestad NK. Motor impairments in young children with cerebral palsy: relationship to gross motor function and everyday activities. Dev Med Child Neurol 2004;46:580-9.

17. Kuijper MA, van der Wilden GJ, Ketelaar M, Gorter JW. Manual ability classification system for children with cerebral palsy in a school setting and its relationship to home self-care activities. Am J Occup Ther
2010;64:614-20.

18. Gunel MK, Mutlu A, Tarsuslu T, Livanelioglu A. Relationship among the Manual Ability Classification System (MACS), the Gross Motor Function Classification System (GMFCS), and the functional status (WeeFIM) in children with spastic cerebral palsy. Eur J Pediatr 2009;168:477-85.

19. Gallahue DL, Ozmun JC. Understanding motor development: infants, children, adolescents, adults. 6th ed. New York: McGraw-Hill; 2006.

20. World Health Organization. International Classification of Functioning, Disability and Health (ICF). Geneva: World Health Organization; 2001.

21. Hauser-Cram P, Warfield ME, Shonkoff JP, Krauss MW, Sayer A, Upshur CC. Children with disabilities: a longitudinal study of child development and parent well-being. Monogr Soc Res Child Dev 2001;66:1-126.

22. Herbert J, Gross J, Hayne H. Crawling is associated with more flexible memory retrieval by 9 -month-old infants. Dev Sci 2007;10:183-9.

23. Rizzolatti G, Matelli M. Two different streams form the dorsal visual system: anatomy and functions. Exp Brain Res 2003;153:146-57. 